\title{
Platinum-Induced Mitochondrial OXPHOS Contributes to Cancer Stem Cell Enrichment in Ovarian Cancer
}

Shruthi Sriramkumar ${ }^{1,2}$, Riddhi Sood ${ }^{2,3}$, Thomas D. Huntington², Ahmed H. Ghobashi' ${ }^{2,3}$,

Weini Wang ${ }^{1,2}$, Kenneth P. Nephew ${ }^{1,2,3,4,5}$, Heather M. O'Hagan ${ }^{1,2,3,4,6}$

${ }^{1}$ Cell, Molecular and Cancer Biology Graduate Program, Indiana University School of Medicine, Bloomington, IN, 47405, USA

${ }^{2}$ Medical Sciences Program, Indiana University School of Medicine, Bloomington, IN, 47405, USA

${ }^{3}$ Genome, Cell and Developmental Biology, Department of Biology, Indiana University Bloomington, Bloomington, IN, 47405, USA

${ }^{4}$ Indiana University Melvin and Bren Simon Comprehensive Cancer Center, Indianapolis, IN, 46202, USA

${ }^{5}$ Department of Anatomy, Cell Biology and Physiology; Department of Obstetrics and Gynecology, Indiana University School of Medicine, Indianapolis, IN, USA

${ }^{6}$ Department of Medical and Molecular Genetics, Indiana University School of Medicine, Indianapolis, IN, USA.

Corresponding Author: Heather M. O'Hagan

Key Words: ALDH+ cells, cancer stem cells, OXPHOS, SIRT1, chemoresistance, ovarian cancer 


\section{Abstract}

\section{Background}

Platinum based agents - cisplatin and carboplatin in combination with taxanes are used for the treatment of ovarian cancer $(\mathrm{OC})$ patients. However, the majority of $\mathrm{OC}$ patients develop recurrent, platinum resistant disease that is uniformly fatal. Acute platinum treatment enriches for chemoresistant aldehyde dehydrogenase (ALDH) + ovarian cancer stem cells (OCSCs), which contribute to tumor recurrence and disease relapse. Acquired platinum resistance includes metabolic reprograming and switching to oxidative phosphorylation (OXPHOS). Chemosensitive cells rely on glycolysis while chemoresistant cells have the ability to switch between glycolysis and OXPHOS, depending on which pathway drives a selective advantage for growth and chemoresistance. High expression of genes involved in OXPHOS and high production of mitochondrial ROS are characteristics of OCSCs, suggesting that OCSCs favor OXPHOS over glycolysis. Based on connections between OCSCs, chemoresistance and OXPHOS, we hypothesize that platinum treatment induces changes in metabolism that contribute to platinum-induced enrichment of OCSCs.

\section{Methods}

The effect of cisplatin on mitochondrial activity was assessed by JC1 staining and expression of OXPHOS genes by quantitative RTPCR. Cisplatin-induced changes in Sirtuin 1 (SIRT1) levels and activity were assessed by Western blot. Small molecule inhibitors of mitochondrial complex I and SIRT1 were used to determine if their enzymatic activity contributes to the platinum-induced enrichment of OCSCs. The 
percentage of ALDH+ OCSCs in OC cells and tumor tissue from xenograft models across different treatment conditions was analyzed using ALDEFLUOR assay and flow cytometry.

\section{Results}

We demonstrate that acute platinum treatment increases mitochondrial activity. Combined treatment of platinum agents and OXPHOS inhibitors blocks the platinuminduced enrichment of ALDH+ OCSCs in vitro and in vivo. Furthermore, platinum treatment increases SIRT1 levels and subsequent deacetylase activity, which likely contributes to the increase in platinum-induced mitochondrial activity.

\section{Conclusions}

These findings on metabolic pathways altered by platinum-based chemotherapy have uncovered key targets that can be exploited therapeutically to block the platinuminduced enrichment of OCSCs, ultimately improving the survival of OC patients. 


\section{Background}

Ovarian cancer $(\mathrm{OC})$ is the $5^{\text {th }}$ leading cause of cancer related deaths among women. The five-year survival rate of OC patients has remained low for decades at $46 \%$ (1). Surgical debulking followed by platinum and taxane-based chemotherapy are standard treatment modalities for OC patients (2). The most common histologic subtype of serous epithelial OC - high grade serous epithelial OC (HGSOC) initially responds well to platinum-based chemotherapy (3). However, eventually patients with HGSOC relapse and develop a chemoresistant disease. Development of platinum resistance is the major obstacle in improving the survival of $\mathrm{OC}$ patients.

In the majority of solid tumors including $\mathrm{OC}$, it has been firmly established that cancer stem cells (CSCs) are responsible for the development of chemoresistance and disease recurrence (4-6). Aldehyde dehydrogenase (ALDH) is the most commonly used robust marker of CSCs (6), and ALDH+ OCSCs have high tumor initiation capacity, enhanced ability to grow as spheroids and express high levels of stemness genes like BMI1, OCT4 and NOTCH3 $(7,8)$. Furthermore, high expression and activity of the ALDH1A isoform strongly correlates with platinum resistant $\mathrm{OC}$ cells (8-10) and negatively correlates with survival of OC patients $(11,12)$. Our group as well as others have demonstrated that acute treatment of $O C$ cells with platinum-based agents results in enrichment of ALDH+ OCSCs $(7,13,14)$. Collectively, the evidence suggests that platinum-induced enrichment of $\mathrm{ALDH}+\mathrm{OCSC}$ contributes to OC recurrence and relapse.

OCSCs are resistant to glucose deprivation and primarily rely on oxidative phosphorylation (OXPHOS) for energy requirements, which may also contribute to 
chemotherapy resistance (15-17). Chemosensitive OC cells favor glycolysis while chemoresistant OC cells are able to switch between glycolysis and OXPHOS depending on which pathway provides selective advantage for growth and platinum resistance (16). OCSCs exhibited high mitochondrial reactive oxygen species (ROS) production and high expression of enzymes involved in OXPHOS, implying that OCSCs preferentially utilize mitochondrial OXPHOS (15). Lung and pancreatic CSCs also displayed higher membrane potential and lower glucose consumption rates compared to non-CSCs $(18,19)$. Thus, there is strong evidence supporting altered metabolism as an important factor driving platinum resistance.

ALDH is a nicotinamide adenine dinucleotide (NAD+) dependent enzyme $(20,21)$. We and others have demonstrated that acute platinum treatment results in an increase in expression of nictotinamide phosphoribosyltransferase (NAMPT) - a rate limiting enzyme in NAD+ biosynthesis salvage pathway. The subsequent increase in cellular $\mathrm{NAD}+$ levels promotes ALDH+ OCSC enrichment and contributes to platinum resistance $(13,14)$. However, it is well-established that platinum resistance is a multifactorial phenomenon (22) and it likely that additional metabolic pathways contribute to the enrichment of ALDH+ OCSCs and chemoresistance.

In the present study, we examined the effect of acute platinum treatment of HGSOC cells on mitochondrial activity. We observed that mitochondrial membrane potential ( $\triangle \boldsymbol{\Psi}_{\mathrm{M}}$ - a surrogate for OXPHOS) and expression of genes involved in mitochondrial OXPHOS increased 16 hours after platinum treatment. Concomitantly, expression of genes in the glycolysis pathway was decreased by acute platinum treatment. Treatment of OC cells with OXPHOS inhibitors blocked the acute platinum- 
induced enrichment of ALDH+ OCSCs, and increased deacetylase activity of sirtuin 1 (SIRT1) was required for platinum-induced enrichment of ALDH+ OCSCs. In addition, we observed that SIRT1-regulated expression of mitochondrial transcription factor A (TFAM) likely contributed to the platinum mediated increase in mitochondrial activity. This first report demonstrating that OXPHOS inhibitors block platinum-induced enrichment of OCSCs supports further investigation into the role of mitochondrial OXPHOS in ovarian tumor recurrence.

\section{Methods}

\section{Cell Culture}

HGSOC cell lines used in the study were maintained at $37^{\circ} \mathrm{C}$ and $5 \% \mathrm{CO}_{2}$ as described previously (23). OVCAR5 and OVSAHO cell lines were authenticated by ATCC in 2018. Briefly, OVCAR5 and OVSAHO cells were cultured in DMEM 1X (Corning, \#MT10013CV) and RPMI 1640 (Corning, \#MT10040CV), respectively, containing 10\% FBS (Gibco, \#16000044) without antibiotics. All the cell lines used in the study were passaged less than 15 times. A $1.67 \mathrm{mM}$ stock solution of cisplatin (Millipore Sigma, \#232120) was made using $154 \mathrm{mM} \mathrm{NaCl}$ (Macron Fine chemicals, \#7581-12) in water. Stock solutions of Rotenone (Sigma, \#R8875-1G; 100 mM), IACS - 010759- OXPHOS Inhibitor (MedChemExpress, \#HY-112037; 10 mM), oligomycin (SelleckChem, \#S1478; 10mM), and SIRT1 inhibitor Ex- 527 (Sigma, E7034; 10 mM) were made in DMSO. For all the experiments using these inhibitors, an equivalent amount of DMSO or inhibitors 
were added along with cisplatin and cells were incubated for 16 hours at $37^{\circ} \mathrm{C}$ and $5 \%$

$\mathrm{CO}_{2}$. All the treatment doses are specified in the figure legends.

\section{JC-1 Staining}

OVCAR5 $\left(1 \times 10^{6}\right)$ and OVSAHO cells $\left(1.5 \times 10^{6}\right)$ were cultured in $100 \mathrm{mM}$ plates for 24 hours and treated with respective $\mathrm{IC}_{50}$ doses of cisplatin (OVCAR5 and OVSAHO 12 $\mu \mathrm{M}$ and $4 \mu \mathrm{M}$, respectively (24)) for 16 hours. JC-1 staining was performed as per the manufacturer's protocol (Thermo Fisher, \#T3168). Briefly, cells were collected and washed with PBS and then resuspended in $1 \mathrm{ml}$ PBS. Stock solutions of JC-1 were made in DMSO at a concentration of $5 \mathrm{mg} / \mathrm{ml}$. Then JC-1 stain was added to cells at a final concentration of $2 \mu \mathrm{g} / \mathrm{ml}$ and cells were incubated at $37^{\circ} \mathrm{C}, 5 \% \mathrm{CO}_{2}$ for 30 minutes. Following incubation, the cells were filtered through $30 \mu \mathrm{m}$ filters and analyzed by flow cytometry.

\section{RNA isolation and quantitative reverse transcription PCR (qRT-PCR)}

Isolation of total RNA from cell pellets was performed using RNAeasy mini kit (Qiagen, \#74104) as per the manufacturer's protocol. Maxima first strand cDNA synthesis kit (Thermo Fisher, \#K1642) for quantitative reverse transcription PCR was used to synthesize cDNA. FastStart Essential DNA green master (Roche, \#06402712001) was used to perform qRT-PCR. Primers of all candidate genes are in Supplemental Table 1. Expression of all the candidate genes were normalized to the housekeeping gene Actin $B$.

\section{ALDEFLUOR assay}


OVCAR5 $\left(1.0 \times 10^{5}\right)$ and OVSAHO $\left(1.5 \times 10^{6}\right)$ were cultured in $100 \mathrm{~mm}$ dishes.

Approximately 24 hours after plating, cells were treated with cisplatin alone or in combination with DMSO or appropriate inhibitors and incubated at $37^{\circ} \mathrm{C}, 5 \% \mathrm{CO}_{2}$ for 16 hours. After the incubation time ALDEFLUOR assay (Stem Cell Technologies, \#01700) was performed as described previously (14).

\section{Flow Cytometry}

LSR II Flow cytometer (BD Biosciences) was used for the analysis of both the JC-1 and ALDEFLUOR assay. JC1 J-monomers and J-aggregates was measured using 488nm excitation and signal was detected using AF488 (green) and PE-A (red), respectively. For all the ALDEFLUOR assays, $488 \mathrm{~nm}$ excitation was used and the signal was detected using 530/30 filter to measure ALDH activity. To determine the percentage of ALDH positive cells in different conditions, the respective DEAB negative control was used. All the data analysis was done using FlowJo software (Becton, Dickinson \& Company).

\section{Generation of stable knockdown lines}

For SIRT1 knockdown (Sigma, NM_012238, TRCN0000018981, TRCN0000018983) and EV TRC1, the lentiviral shRNA knockdown protocol from the RNAi consortium of the Broad Institute was used as described previously (23).

\section{Mitochondrial DNA (mtDNA) content analysis}

Mitochondrial DNA content was quantified relative to nuclear DNA (nDNA) ( $\beta_{2}-$ microglobulin) using qPCR and Delta Ct method as described previously (25-27). See Supplementary Table 1 for primers. 


\section{Xenograft studies}

All the animal studies were performed in accordance with the Association for Assessment and Accreditation of Laboratory Animal Care International and approved by Indiana University Bloomington Institutional Animal Care and Use Committee as described previously (14). $2 \times 10^{6}$ OVCAR3 cells were injected subcutaneously in the flanks of NSG mice (IUSCCC In Vivo Therapeutics Core). Once the established tumors reached $>100 \mathrm{~mm}^{3}$, they were randomized into 3 different treatment groups and treated with vehicle alone or combination of carboplatin and vehicle or IACS-010759 (OXPHOS inhibitor). Carboplatin was administered once a week intraperitoneally at $50 \mathrm{mg} / \mathrm{kg}$ for 3 weeks. IACS-010759 was prepared in 10\% DMSO and 90\% corn oil. IACS-010759 was administered orally at $7.5 \mathrm{mg} / \mathrm{kg}$ five days a week for 3 weeks. At the end of the treatment schedule, mice were sacrificed and tumors were collected, disassociated and used for ALDEFLUOR assay as we have done previously $(7,14)$. Tumor dissociation kit and gentle MACS dissociator (Miltenyi Biotech) was used to dissociate the tumors into single cells as per manufacturer's protocol.

\section{Correlation analysis using cBioportal}

Correlation analysis of expression of SIRT1 and TFAM was performed using the cBioportal $(28,29)$ and RNA-seq data of ovarian cancer samples from The Cancer Genome Atlas (30).

\section{Antibodies}

For western blot, the following antibodies were used: anti-pATMS1981 (Cell Signaling Technology (CST), \#13050, 1:1000), anti-AcH4K16 (CST, \#13534, 1:1000), anti-total 
H2A (CST, \#12349, 1:1000), anti-SIRT1 (Santa Cruz, sc-74465, 1:1000) and antivimentin (CST, \#5741).

\section{Statistics}

The percentage of ALDH+ cells across different conditions after ALDEFLUOR assay was evaluated by one-way ANOVA with multiple comparisons using Graphpad prism and data is presented as \pm SEM. Data of JC- 1 staining, RT-qPCR and densitometry were evaluated by Student $t$ test in Graphpad prism and excel.

\section{Results}

\section{Acute platinum treatment increases mitochondrial OXPHOS activity}

Development of platinum resistance in $\mathrm{OC}$ has been linked to increased reliance of cancer cells on OXPHOS (31). Therefore, first we determined if mitochondrial membrane potential ( $\triangle \boldsymbol{\Psi}_{\mathrm{M}}$ - a surrogate for OXPHOS)(32) increased after treatment of OC cells with cisplatin. To measure $\Delta \Psi_{\mathrm{M}}$, a lipophilic, cationic dye called JC-1 was used. JC-1 enters the mitochondria of healthy cells with normal $\Delta \Psi_{M}$ and spontaneously aggregates as red fluorescent J-aggregates; in contrast, in cells with disrupted $\Delta \boldsymbol{\Psi}_{\mathrm{M}}, \mathrm{JC}$ 1 will not form aggregates and retains its green fluorescence (32). Treatment of HGSOC cells - OVCAR5, OVSAHO with their respective $\mathrm{IC}_{50}$ doses of cisplatin for 16 hours followed by JC-1 staining resulted in a shift in the population of cells from low red fluorescence to high red fluorescence (Figure 1A,B; Supplementary Figure S1A), indicating an increase in mitochondrial J-aggregates after acute cisplatin treatment. A histogram of the red fluorescence for $\mathrm{JC}-1$ staining in untreated and cisplatin treated 
OVCAR5 and OVSAHO cells confirmed that cisplatin treated cells shifted towards higher red fluorescence (Figure 1C, D). Furthermore, the ratio of red to green fluorescence significantly increased after acute cisplatin treatment of both OVCAR5 and OVSAHO cells (Figure 1E, F). Treatment of OVCAR5 cells with cisplatin for 16 hours had no effect on the mitochondrial DNA (mtDNA) content relative to nuclear DNA content (Supplementary Figure S1B), further supporting that the cisplatin-induced increase in OXPHOS was due to an increase in mitochondrial activity, not an increase in mitochondrial number.

\section{Acute cisplatin treatment induces OXPHOS gene expression}

To determine if acute cisplatin treatment increased expression of genes of mitochondrial OXPHOS complexes, we performed qRT-PCR of untreated and 16 hour cisplatin-treated cells. Increased expression of Complex I subunits - NADH ubiquinone oxidoreductase subunits S6, A11 (NDUFS6, NDUFA11); Complex III subunits ubiquinol-cytochrome C reductase subunit X, XI (UQCR10, UQCR11); Cytochrome C oxidase complex subunits 5A, 6A (COX5A, COX6A); ATP synthase membrane subunit F (ATP5MF) and translocase of the inner mitochondrial membrane (TIMM17A) was observed in both OVCAR5 and OVSAHO cells after acute cisplatin treatment (Figure 2A, B). In addition, we examined genes associated with hexokinase, which is involved in the first step in glycolysis (33), as well as hypoxia-inducible factor 1 (HIF-1), known to inhibit mitochondrial biogenesis and respiration by negatively regulating the activity of transcription factor c-Myc $(34,35)$. Treatment of OVCAR5 and OVSAHO cells with cisplatin for 16 hours decreased expression of hexokinase isoforms hexokinase $1(H K 1)$ and hexokinase 2 (HK2) (Figure 2C, 2D) as well as HIF1a (Figure 2E) and increased $c^{-}$ 
Myc expression (Figure 2F). Altogether, our findings suggest that acute platinum treatment caused an increase in $\Delta \Psi_{M}$ and OXPHOS.

\section{OXPHOS is essential for the platinum-induced enrichment of ALDH+ cells}

Acute platinum treatment has been previously demonstrated to enrich the ALDH+ OCSC population $(7,13,14)$. To determine if the increase in OXPHOS in response to acute cisplatin treatment is essential for the platinum-induced enrichment of ALDH+ cells, we treated OVCAR5 and OVSAHO cells with cisplatin alone or in combination with $5 \mu \mathrm{M}$ Rotenone, a mitochondrial complex I inhibitor, or DMSO and performed the ALDEFLUOR assay. As expected, treatment of OVCAR5 and OVSAHO cells with cisplatin alone or in combination with DMSO increased the percentage of ALDH+ OCSCs (Figure 3A, B). Rotenone treatment blocked the platinum-induced increase in \%ALDH+ OCSCs without changing the basal percentage of ALDH+ cells. IACS 010759 is a novel small molecule OXPHOS inhibitor, which like rotenone also inhibits complex I of the mitochondrial respiratory electron transport chain (36). Treatment of OVCAR5 and OVSAHO cells with $1 \mu \mathrm{M}$ IACS-010759 in combination with cisplatin abrogated the platinum-induced enrichment of ALDH+ cells (Figure $3 \mathrm{C}-\mathrm{E}$;

Supplementary Figure S2A, S2B). Oligomycin inhibits the ATP synthase complex in the mitochondrial respiratory electron transport chain (37), and combination treatment of 1 $\mu \mathrm{M}$ oligomycin plus cisplatin blocked the platinum-induced increase in ALDH+cells (Figure 3F). Altogether, these data suggest that the increase in mitochondrial activity observed after acute cisplatin treatment is required for the platinum-induced enrichment of $\mathrm{ALDH}+\mathrm{OCSCs}$ 


\section{SIRT1 contributes to the platinum-induced enrichment of ALDH+ cells}

SIRT1 deacetylates acetylated histone 4 lysine 16 (H4K16ac) as well as nonhistone proteins and has been implicated in the regulation of metabolic processes (38, 39). Cellular NAD+ levels were reported to regulate the deacetylase activity of SIRT1 after acute platinum treatment $(13,14,40)$, and SIRT1 expression and activity was also increased in platinum resistant $\mathrm{OC}$ cells $(41,42)$. However, the precise mechanism by which SIRT1 contributes to platinum resistance remains incompletely understood. Cisplatin treatment for 16 hours reduced the levels of $\mathrm{H} 4 \mathrm{~K} 16 \mathrm{ac}$ relative to untreated OVCAR5 cells (Figure 4A). Phosphorylation of ATM (p-ATM) at serine 1981 was used as a control for platinum-induced DNA damage response and we observed an expected increase in ATM phosphorylation at S1981 after platinum treatment in OVCAR5 (Figure 4A). Treatment of OVCAR5 and OVSAHO cells with the IC50 dose of cisplatin for 16 hours also resulted in increased mRNA and protein levels of SIRT1 (Figure 4B-D). Furthermore, SIRT1 knockdown (KD) using shRNA rescued the platinum-induced decrease in H4K16Ac (Figure 4D), confirming that SIRT1 deacetylates H4K16 in response to acute cisplatin treatment. KD of SIRT1 using two different shRNAs in OVCAR5 cells followed by cisplatin treatment blocked the platinum-induced increase in $\mathrm{ALDH}+$ cells and the baseline percentage of ALDH+ cells remained unchanged (Figure 4E). To further investigate if inhibiting SIRT1 would block platinum-induced enrichment of ALDH+ cells, OVCAR5 and OVSAHO cells were treated with cisplatin alone or cisplatin plus SIRT1 inhibitor Ex-527 (3 $\mu \mathrm{M})$ or DMSO followed by ALDEFLUOR assay. In both OC cell lines, inhibiting SIRT1 completely abrogated the platinum-induced increase in ALDH+ cells (Figure 4F, G). 
SIRT1 has been shown to promote OXPHOS and mitochondrial biogenesis in adipocytes and hepatocellular carcinoma cells by regulating the expression of mitochondrial transcription factor A (TFAM) (43-45). TFAM is important for proper transcription of mitochondrial and OXPHOS genes $(46,47)$. To test the hypothesis that SIRT1 promoted the platinum-induced increase in ALDH+ cells by promoting the increase in mitochondrial OXPHOS, we first analyzed the correlation between SIRT1 and TFAM expression in publicly available RNA-sequencing datasets from OC patients (30). SIRT1 expression was positively correlated with TFAM expression (Figure 4H). Next, we determined if the expression of TFAM is dependent on SIRT1 in response to acute cisplatin treatment in OC cells. SIRT1 KD using two different shRNAs resulted in a modest but significant reduction in the platinum-induced increase in TFAM expression (Figure 4I). Altogether, these data suggest that SIRT1 activity plays a key role in platinum-induced enrichment of $\mathrm{ALDH}+$ cells in $\mathrm{OC}$.

\section{OXPHOS inhibition blocks the platinum-induced enrichment of ALDH+ cells in} vivo

We and others have demonstrated that acute platinum treatment results in enrichment of ALDH+ OCSCs in vitro and in vivo $(7,13,48,49)$. Furthermore, we demonstrated that treatment of OC cells with OXPHOS inhibitors in combination with cisplatin blocked the platinum-induced enrichment of ALDH+ cells in vitro without affecting the basal ALDH percentage (Figure 3A-F). Therefore, it was of interest to determine if OXPHOS inhibition blocks the platinum mediated increase in ALDH+ OCSCs in vivo in mouse xenografts. As shown in Figure 5A, 2 million OVCAR3 cells were injected subcutaneously in the flanks of NSG mice and after establishment of 
tumors, randomized mice received vehicle alone or a combination of carboplatin + vehicle or OXPHOS inhibitor IACS-010759. As expected, platinum treatment significantly reduced the tumor volume compared to the vehicle only group (Figure 5B). In the group treated with carboplatin + IACS-010759, tumor volume was less than the group treated with carboplatin alone at the end of the study (Figure 5B). We observed the expected increase in the percentage of ALDH+ cells in the carboplatin + vehicle compared to the vehicle only group, consistent with previous studies by us and others $(7,13,48,49)$. The combination treatment of IACS-010759 + carboplatin abrogated the platinum-induced enrichment of $\mathrm{ALDH}+$ cells in vivo (Figure $5 \mathrm{C}$ ). Altogether, we demonstrate that inhibiting OXPHOS with a complex I mitochondrial inhibitor blocks platinum-induced enrichment of $\mathrm{ALDH}+$ cells in vivo.

\section{Discussion}

Metabolic reprogramming is a distinctive characteristic of chemoresistant OC cells and likely occurs as a consequence of metabolic adaptation to the tumor microenvironment (16). Metabolic reprogramming provides adenosine triphosphate (ATP) and precursors for macromolecular biosynthesis to meet the energy requirements for rapid proliferation and survival of tumor cells (50). In addition to promoting tumorigenesis, alterations in cellular metabolism foster resistance to chemotherapy in $\mathrm{OC}$ and other cancer types (51-53). The predominant mechanism of cytotoxicity of chemotherapeutic agents is inducing DNA damage and oxidative stress. Metabolic reprogramming enables tumor cells to adapt and manage pharmacological insults and oxidative stress (50). The precise mechanism of how metabolic reprogramming 
promotes chemoresistance in $\mathrm{OC}$ is not understood. Several studies have established that $\mathrm{ALDH}+\mathrm{CSC}$ contribute to the development of chemoresistance in $\mathrm{OC}(7,13,14)$. Here, we demonstrate for the first time a role for OXPHOS and SIRT1 in the platinuminduced enrichment of ALDH+ OCSCs.

There has been a long-standing debate about whether CSCs use glycolysis or OXPHOS for survival (54). The metabolic pathway utilized by CSCs depends on which pathway provides a selective advantage in the tumor microenvironment. Here, we demonstrate that acute cisplatin treatment elevates mitochondrial OXPHOS concurrently with an increase in percentage of ALDH+ cells. Furthermore, OXPHOS inhibitors in combination with cisplatin block the enrichment of ALDH+ cells after acute cisplatin treatment, suggesting that the platinum mediated increase in mitochondrial activity is required for the enrichment of $A L D H+$ cells. These findings agree with Viale et al. that pancreatic CSCs depend on OXPHOS for survival $(19,55)$. It is likely that OXPHOS generates ROS to regulate metabolic plasticity of OCSCs, as recently suggested by Luo and Wicha (56).

Platinum resistant OC cells display elevated expression of the deacetylase SIRT1 $(41,42)$. We demonstrate that SIRT1 levels and activity increase after acute platinum treatment. NAD+ functions as a co-factor for several enzymes like ALDH and SIRT1 $(21,57)$, and the increase in SIRT1 activity and expression in response to acute cisplatin treatment may be due to platinum-induced increase in cellular NAD+ levels (13, 14). The dependency of the platinum mediated increase in ALDH+ cells on SIRT1 may be due to enhanced regulation of the platinum-induced metabolic switch to oxidative metabolism and improved antioxidant defenses by SIRT1, both of which could promote 
survival of CSCs $(40,58)$. SIRT1 supports mitochondrial function in muscle cells after exercise or starvation by regulating $\operatorname{TFAM}(59,60)$, and we show that SIRT1 KD reduces the platinum-induced increase in TFAM expression. However, the precise mechanism of how SIRT1 contributes to the increase in ALDH+ cells and regulates TFAM in response to acute cisplatin treatment requires further investigation.

\section{Conclusions}

The persistence of ALDH+ cells after chemotherapy is a major cause of disease recurrence and relapse in OC (4). We have demonstrated that acute cisplatin treatment of $\mathrm{OC}$ cells results in an increase in mitochondrial membrane potential. Our in vitro and in vivo data demonstrate that combination treatment with an OXPHOS inhibitor and platinum blocks the platinum-induced enrichment of ALDH+ cells. Our findings reinforce the need for additional preclinical and clinical investigations aimed at exploiting OXPHOS inhibitors to delay tumor recurrence and improve survival in OC.

\section{List of Abbreviations}

$\Delta \Psi_{\mathrm{M}} \quad$ Mitochondrial membrane potential

ALDH Aldehyde dehydrogenase

ATM Ataxia telangiectasia mutated

ATP5MF ATP synthase membrane subunit $F$

COX5A Cytochrome C oxidase subunit 5A 
COX6A Cytochrome $\mathrm{C}$ oxidase subunit 6A

CSCs Cancer stem cells

DMSO Dimethyl sulfoxide

HGSOC High grade serous ovarian cancer

HIF1 $\quad$ Hypoxia-inducible factor 1

HK1 Hexokinase 1

HK2 Hexokinase 2

KD Knockdown

mtDNA Mitochondrial DNA

NAD+ Nicotinamide adenine dinucleotide

NAMPT Nicotinamide phosphoribosyltransferase

NDUFA11 NADH ubiquinone oxidoreductase subunit 11

NDUFS6 NADH ubiquinone oxidoreductase subunit 6

OC Ovarian Cancer

OcSCs Ovarian Cancer Stem Cells

OXPHOS Oxidative phosphorylation

qRT-PCR quantitative reverse transcriptase polymerase chain reaction

ROS Reactive oxygen species

shRNA short hairpin RNA

SIRT1 Sirtuin 1 
TFAM Mitochondrial transcription factor A

TIMM17A Translocase of the inner mitochondrial membrane

UQCR10 Ubiquinol- cytochrome $\mathrm{C}$ reductase subunit $\mathrm{X}$

UQCR11 Ubiquinol-cytochrome C reductase subunit XI

\section{Declarations}

Ethics approval and consent to participate

The animal study was approved by Indiana University Bloomington Institutional Animal

Care and Use Committee and was performed in adherence with the Association for

Assessment and Accreditation of Laboratory Animal Care International.

\section{Consent for publication}

Not applicable

\section{Availability of data and materials}

All the data generated in the manuscript and materials used in the study are available upon reasonable request.

\section{Competing Interests}

The authors declare no competing interests. 


\section{Funding}

This research was funded in part by the Ovarian Cancer Research Alliance (grant number 458788 to $\mathrm{HMOH}$ and KPN) and through the IU Simon Comprehensive Cancer Center P30 Support Grant (P30CA082709). SS was supported by the Doane and Eunice Dahl Wright Fellowship generously provided by Ms. Imogen Dahl.

\section{Author Contributions}

SS conceived, designed, analyzed all the experiments, wrote the original draft of the manuscript, edited and proofread the manuscript. RS, TDH and WW assisted in data acquisition. AHG performed some validation experiments and proofread the manuscript. KPN provided resources, funding acquisition, edited and proofread the manuscript. $\mathrm{HMOH}$ conceived, assisted with experimental design, supervised the study, and provided resources, funding acquisition, edited and proofread the manuscript. All authors have approved the submitted version of the manuscript.

\section{Acknowledgments}

We thank Christiane Hassel and the Indiana University Flow Cytometry facility for their assistance. 


\section{References}

1. Torre LA, Trabert B, DeSantis CE, Miller KD, Samimi G, Runowicz CD, et al. Ovarian cancer statistics, 2018. CA Cancer J Clin. 2018;68(4):284-96.

2. Ozols RF. Treatment goals in ovarian cancer. Int J Gynecol Cancer. 2005;15 Suppl 1:3-11.

3. Vaughan S, Coward JI, Bast RC, Jr., Berchuck A, Berek JS, Brenton JD, et al. Rethinking ovarian cancer: recommendations for improving outcomes. Nat Rev Cancer. 2011;11(10):719-25.

4. Lupia M, Cavallaro U. Ovarian cancer stem cells: still an elusive entity? Mol Cancer. 2017;16(1):64.

5. Gupta PB, Chaffer CL, Weinberg RA. Cancer stem cells: mirage or reality? Nat Med. 2009;15(9):1010-2.

6. Muralikrishnan V, Hurley TD, Nephew KP. Targeting Aldehyde Dehydrogenases to Eliminate Cancer Stem Cells in Gynecologic Malignancies. Cancers (Basel). 2020;12(4).

7. Wang Y, Cardenas H, Fang F, Condello S, Taverna P, Segar M, et al. Epigenetic targeting of ovarian cancer stem cells. Cancer Res. 2014;74(17):4922-36.

8. Landen CN, Jr., Goodman B, Katre AA, Steg AD, Nick AM, Stone RL, et al. Targeting aldehyde dehydrogenase cancer stem cells in ovarian cancer. Mol Cancer Ther. 2010;9(12):3186-99.

9. $\quad$ Condello S, Morgan CA, Nagdas S, Cao L, Turek J, Hurley TD, et al. betaCatenin-regulated ALDH1A1 is a target in ovarian cancer spheroids. Oncogene. 2015;34(18):2297-308.

10. Januchowski R, Wojtowicz K, Sterzyska K, Sosiska P, Andrzejewska M, Zawierucha $\mathrm{P}$, et al. Inhibition of ALDH1A1 activity decreases expression of drug transporters and reduces chemotherapy resistance in ovarian cancer cell lines. Int $\mathrm{J}$ Biochem Cell Biol. 2016;78:248-59.

11. Silva IA, Bai S, McLean K, Yang K, Griffith K, Thomas D, et al. Aldehyde dehydrogenase in combination with CD133 defines angiogenic ovarian cancer stem cells that portend poor patient survival. Cancer Res. 2011;71(11):3991-4001.

12. Kuroda T, Hirohashi Y, Torigoe T, Yasuda K, Takahashi A, Asanuma H, et al. ALDH1-high ovarian cancer stem-like cells can be isolated from serous and clear cell adenocarcinoma cells, and ALDH1 high expression is associated with poor prognosis. PLoS One. 2013;8(6):e65158.

13. Nacarelli T, Fukumoto T, Zundell JA, Fatkhutdinov N, Jean S, Cadungog MG, et al. NAMPT Inhibition Suppresses Cancer Stem-like Cells Associated with TherapyInduced Senescence in Ovarian Cancer. Cancer Res. 2020;80(4):890-900.

14. Sood R, ; Xiao, S,; Sriramkumar, S,; Hassel, C,; Nephew, K.P,; O'Hagan, H.M. Novel epigenetic-metabolic inhibitor combination treatment blocks platinum-induced ovarian cancer stem cell enrichment. Biorxiv. 2021.

15. Pasto A, Bellio C, Pilotto G, Ciminale V, Silic-Benussi M, Guzzo G, et al. Cancer stem cells from epithelial ovarian cancer patients privilege oxidative phosphorylation, and resist glucose deprivation. Oncotarget. 2014;5(12):4305-19. 
16. Dar S, Chhina J, Mert I, Chitale D, Buekers T, Kaur H, et al. Bioenergetic Adaptations in Chemoresistant Ovarian Cancer Cells. Sci Rep. 2017;7(1):8760.

17. Ghoneum A, Gonzalez D, Abdulfattah AY, Said N. Metabolic Plasticity in Ovarian Cancer Stem Cells. Cancers (Basel). 2020;12(5).

18. Ye XQ, Li Q, Wang GH, Sun FF, Huang GJ, Bian XW, et al. Mitochondrial and energy metabolism-related properties as novel indicators of lung cancer stem cells. Int $\mathrm{J}$ Cancer. 2011;129(4):820-31.

19. Viale A, Pettazzoni P, Lyssiotis CA, Ying H, Sanchez N, Marchesini M, et al. Oncogene ablation-resistant pancreatic cancer cells depend on mitochondrial function. Nature. 2014;514(7524):628-32.

20. Kiefer FW, Orasanu G, Nallamshetty S, Brown JD, Wang H, Luger P, et al. Retinaldehyde dehydrogenase 1 coordinates hepatic gluconeogenesis and lipid metabolism. Endocrinology. 2012;153(7):3089-99.

21. Chen Y, Thompson DC, Koppaka V, Jester JV, Vasiliou V. Ocular aldehyde dehydrogenases: protection against ultraviolet damage and maintenance of transparency for vision. Prog Retin Eye Res. 2013;33:28-39.

22. Stewart DJ. Mechanisms of resistance to cisplatin and carboplatin. Crit Rev Oncol Hematol. 2007;63(1):12-31.

23. Sriramkumar S, Matthews TD, Ghobashi AH, Miller SA, VanderVere-Carozza PS, Pawelczak KS, et al. Platinum-Induced Ubiquitination of Phosphorylated H2AX by RING1A Is Mediated by Replication Protein A in Ovarian Cancer. Mol Cancer Res. 2020;18(11):1699-710.

24. Haley J, Tomar S, Pulliam N, Xiong S, Perkins SM, Karpf AR, et al. Functional characterization of a panel of high-grade serous ovarian cancer cell lines as representative experimental models of the disease. Oncotarget. 2016;7(22):32810-20. 25. Maiuri AR, Li H, Stein BD, Tennessen JM, O'Hagan HM. Inflammation-induced DNA methylation of DNA polymerase gamma alters the metabolic profile of colon tumors. Cancer Metab. 2018;6:9.

26. Rooney JP, Ryde IT, Sanders LH, Howlett EH, Colton MD, Germ KE, et al. PCR based determination of mitochondrial DNA copy number in multiple species. Methods Mol Biol. 2015;1241:23-38.

27. Machado TS, Macabelli CH, Sangalli JR, Rodrigues TB, Smith LC, Meirelles FV, et al. Real-Time PCR Quantification of Heteroplasmy in a Mouse Model with Mitochondrial DNA of C57BL/6 and NZB/BINJ Strains. PLoS One. 2015;10(8):e0133650.

28. Cerami E, Gao J, Dogrusoz U, Gross BE, Sumer SO, Aksoy BA, et al. The cBio cancer genomics portal: an open platform for exploring multidimensional cancer genomics data. Cancer Discov. 2012;2(5):401-4.

29. Gao J, Aksoy BA, Dogrusoz U, Dresdner G, Gross B, Sumer SO, et al. Integrative analysis of complex cancer genomics and clinical profiles using the cBioPortal. Sci Signal. 2013;6(269):pl1.

30. Cancer Genome Atlas Research N. Integrated genomic analyses of ovarian carcinoma. Nature. 2011;474(7353):609-15.

31. Matassa DS, Amoroso MR, Lu H, Avolio R, Arzeni D, Procaccini C, et al. Oxidative metabolism drives inflammation-induced platinum resistance in human ovarian cancer. Cell Death Differ. 2016;23(9):1542-54. 
32. Sivandzade F, Bhalerao A, Cucullo L. Analysis of the Mitochondrial Membrane Potential Using the Cationic JC-1 Dye as a Sensitive Fluorescent Probe. Bio Protoc. 2019;9(1).

33. DeWaal D, Nogueira V, Terry AR, Patra KC, Jeon SM, Guzman G, et al. Hexokinase-2 depletion inhibits glycolysis and induces oxidative phosphorylation in hepatocellular carcinoma and sensitizes to metformin. Nat Commun. 2018;9(1):446. 34. Zhang H, Gao P, Fukuda R, Kumar G, Krishnamachary B, Zeller KI, et al. HIF-1 inhibits mitochondrial biogenesis and cellular respiration in VHL-deficient renal cell carcinoma by repression of C-MYC activity. Cancer Cell. 2007;11(5):407-20.

35. Sutphin PD, Giaccia AJ, Chan DA. Energy regulation: HIF MXles it up with the CMYC powerhouse. Dev Cell. 2007;12(6):845-6.

36. Molina JR, Sun Y, Protopopova M, Gera S, Bandi M, Bristow C, et al. An inhibitor of oxidative phosphorylation exploits cancer vulnerability. Nat Med. 2018;24(7):1036-46. 37. Ashton TM, McKenna WG, Kunz-Schughart LA, Higgins GS. Oxidative Phosphorylation as an Emerging Target in Cancer Therapy. Clin Cancer Res. 2018;24(11):2482-90.

38. Liu TF, McCall CE. Deacetylation by SIRT1 Reprograms Inflammation and Cancer. Genes Cancer. 2013;4(3-4):135-47.

39. Vaquero A, Scher M, Lee D, Erdjument-Bromage H, Tempst P, Reinberg D. Human SirT1 interacts with histone $\mathrm{H} 1$ and promotes formation of facultative heterochromatin. Mol Cell. 2004;16(1):93-105.

40. Canto C, Auwerx J. Targeting sirtuin 1 to improve metabolism: all you need is NAD(+)? Pharmacol Rev. 2012;64(1):166-87.

41. Imai S, Armstrong CM, Kaeberlein M, Guarente L. Transcriptional silencing and longevity protein Sir2 is an NAD-dependent histone deacetylase. Nature. 2000;403(6771):795-800.

42. Liang XJ, Finkel T, Shen DW, Yin JJ, Aszalos A, Gottesman MM. SIRT1 contributes in part to cisplatin resistance in cancer cells by altering mitochondrial metabolism. Mol Cancer Res. 2008;6(9):1499-506.

43. Li Y, Xu S, Li J, Zheng L, Feng M, Wang X, et al. SIRT1 facilitates hepatocellular carcinoma metastasis by promoting PGC-1alpha-mediated mitochondrial biogenesis. Oncotarget. 2016;7(20):29255-74.

44. Majeed $\mathrm{Y}$, Halabi N, Madani AY, Engelke R, Bhagwat AM, Abdesselem $\mathrm{H}$, et al. SIRT1 promotes lipid metabolism and mitochondrial biogenesis in adipocytes and coordinates adipogenesis by targeting key enzymatic pathways. Sci Rep. 2021;11(1):8177.

45. Zhang J, Peng J, Kong D, Wang X, Wang Z, Liu J, et al. Silent information regulator 1 suppresses epithelial-to-mesenchymal transition in lung cancer cells via its regulation of mitochondria status. Life Sci. 2021;280:119716.

46. Kaufman BA, Durisic N, Mativetsky JM, Costantino S, Hancock MA, Grutter P, et al. The mitochondrial transcription factor TFAM coordinates the assembly of multiple DNA molecules into nucleoid-like structures. Mol Biol Cell. 2007;18(9):3225-36.

47. Kanki T, Ohgaki K, Gaspari M, Gustafsson CM, Fukuoh A, Sasaki N, et al. Architectural role of mitochondrial transcription factor $A$ in maintenance of human mitochondrial DNA. Mol Cell Biol. 2004;24(22):9823-34. 
48. Wang Y, Zong X, Mitra S, Mitra AK, Matei D, Nephew KP. IL-6 mediates platinum-induced enrichment of ovarian cancer stem cells. JCI Insight. 2018;3(23).

49. $\quad$ Wang Y, Zhao G, Condello S, Huang H, Cardenas H, Tanner EJ, et al. Frizzled-7 Identifies Platinum-Tolerant Ovarian Cancer Cells Susceptible to Ferroptosis. Cancer Res. 2021;81(2):384-99.

50. Chen X, Chen S, Yu D. Metabolic Reprogramming of Chemoresistant Cancer Cells and the Potential Significance of Metabolic Regulation in the Reversal of Cancer Chemoresistance. Metabolites. 2020;10(7).

51. Han CY, Patten DA, Richardson RB, Harper ME, Tsang BK. Tumor metabolism regulating chemosensitivity in ovarian cancer. Genes Cancer. 2018;9(5-6):155-75.

52. Montopoli M, Bellanda M, Lonardoni F, Ragazzi E, Dorigo P, Froldi G, et al. "Metabolic reprogramming" in ovarian cancer cells resistant to cisplatin. Curr Cancer Drug Targets. 2011;11(2):226-35.

53. Cardoso MR, Santos JC, Ribeiro ML, Talarico MCR, Viana LR, Derchain SFM. A Metabolomic Approach to Predict Breast Cancer Behavior and Chemotherapy Response. Int J Mol Sci. 2018;19(2).

54. Peiris-Pages M, Martinez-Outschoorn UE, Pestell RG, Sotgia F, Lisanti MP. Cancer stem cell metabolism. Breast Cancer Res. 2016;18(1):55.

55. Viale A, Corti D, Draetta GF. Tumors and mitochondrial respiration: a neglected connection. Cancer Res. 2015;75(18):3685-6.

56. Luo M, Wicha MS. Targeting Cancer Stem Cell Redox Metabolism to Enhance Therapy Responses. Semin Radiat Oncol. 2019;29(1):42-54.

57. Guo X, Kesimer M, Tolun G, Zheng X, Xu Q, Lu J, et al. The NAD(+)-dependent protein deacetylase activity of SIRT1 is regulated by its oligomeric status. Sci Rep. 2012;2:640.

58. Singh CK, Chhabra G, Ndiaye MA, Garcia-Peterson LM, Mack NJ, Ahmad N. The Role of Sirtuins in Antioxidant and Redox Signaling. Antioxid Redox Signal. 2018;28(8):643-61.

59. Chandrasekaran K, Anjaneyulu M, Choi J, Kumar P, Salimian M, Ho CY, et al. Role of mitochondria in diabetic peripheral neuropathy: Influencing the NAD(+)dependent SIRT1-PGC-1alpha-TFAM pathway. Int Rev Neurobiol. 2019;145:177-209. 60. Ruderman NB, Xu XJ, Nelson L, Cacicedo JM, Saha AK, Lan F, et al. AMPK and SIRT1: a long-standing partnership? Am J Physiol Endocrinol Metab. 2010;298(4):E751-60. 


\section{Figures}

A.

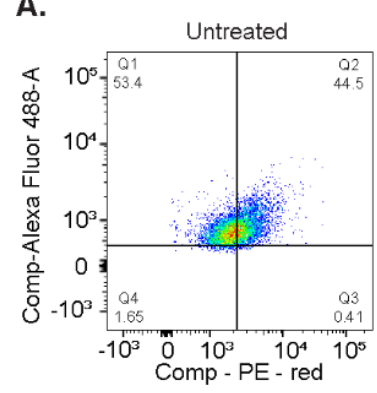

C.

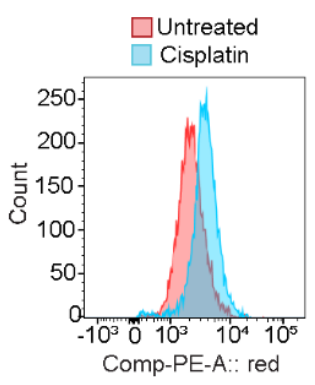

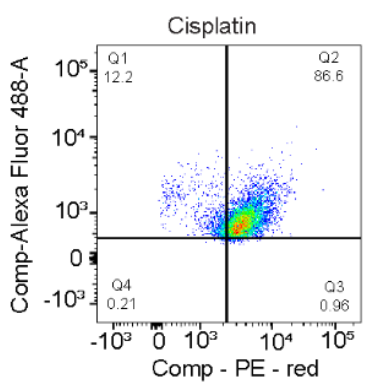

D.

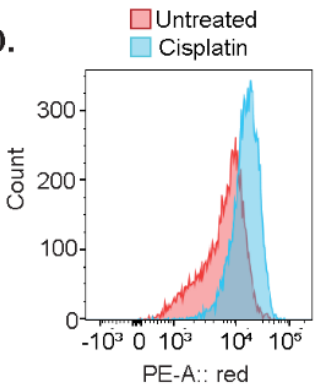

B.
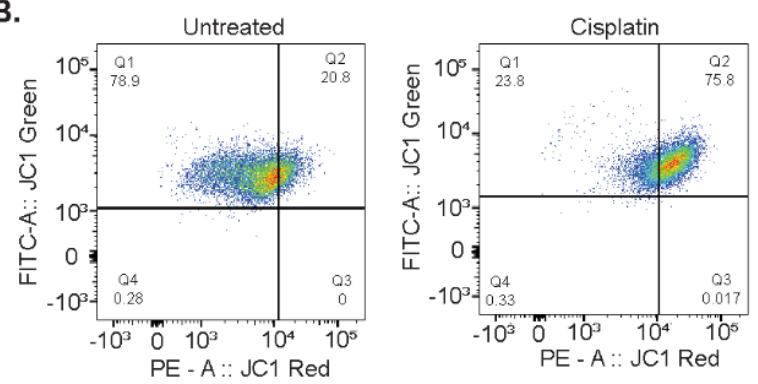

E.

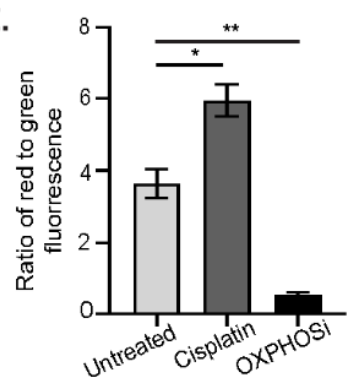

F.

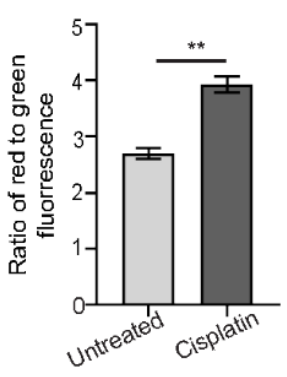

Figure 1. Mitochondrial OXPHOS activity increases in response to acute cisplatin treatment in OC cells. Scatter plots of OVCAR5 (A) and OVSAHO (B) cells untreated or treated with $12 \mu \mathrm{M}$ and $4 \mu \mathrm{M}$ cisplatin, respectively, for 16 hours followed by staining with $\mathrm{JC}-1$ for 30 minutes and analysis by flow cytometry. Histogram of untreated and cisplatin treated OVCAR5 (C) and OVSAHO (D) cells after JC-1 staining as in A and B. Ratio of red to green fluorescence intensity in untreated and cisplatin treated OVCAR5 (E) and OVSAHO (F) cells after JC-1 staining as in A and B. The OXPHOS inhibitor carbonyl cyanide 3-chlorophenylhydrazone (OXPHOSi) was used as a control. Graphs display mean ratio of red to green fluorescence \pm SEM $(N=3)$. Student t-test was used to calculate statistical significance. For all untreated versus cisplatin treated, $P$ values ${ }^{*}<$ $0.05,{ }^{* *}<0.005$ and ${ }^{* * *}<0.0005$. 
A.

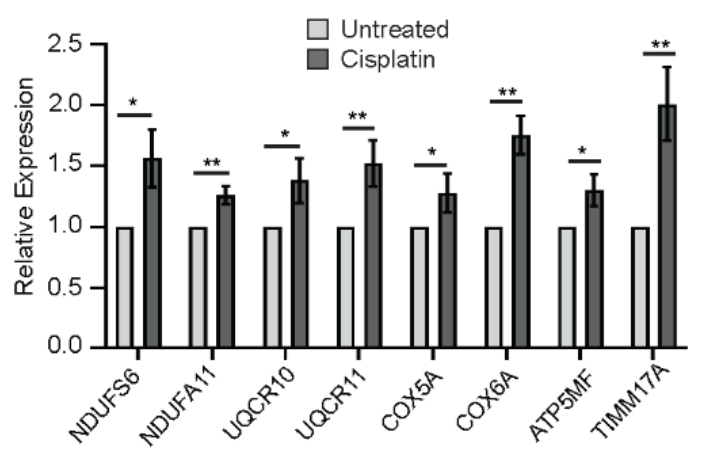

C.

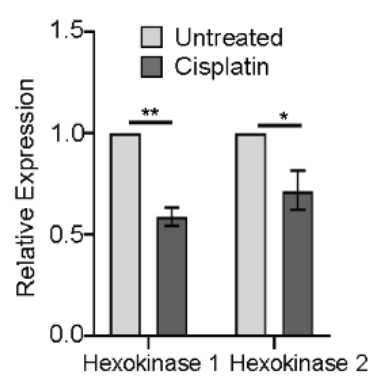

D.

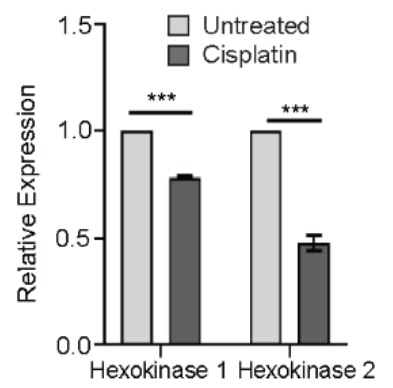

B.
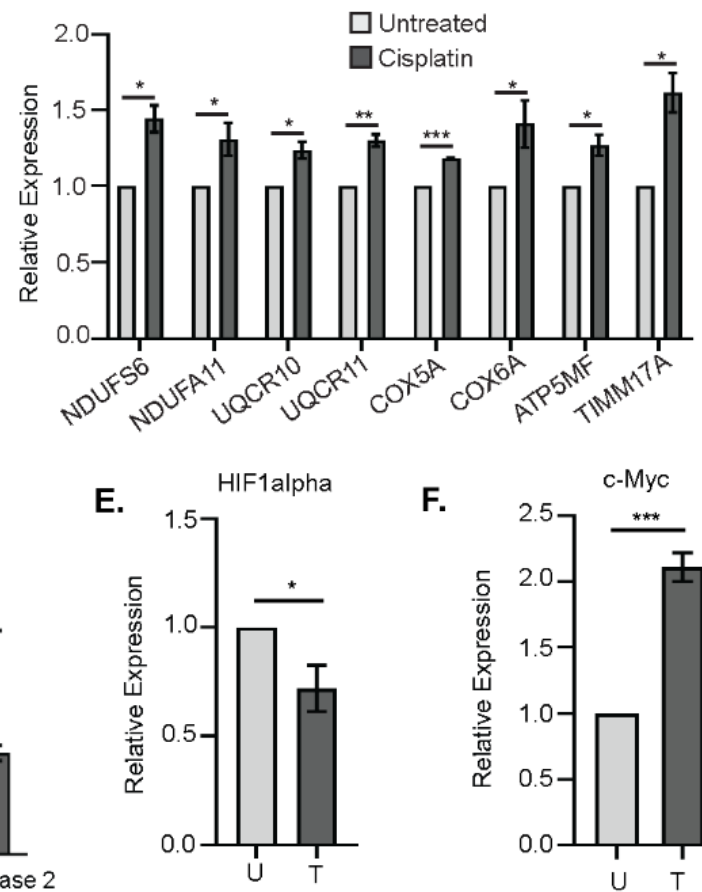

F.

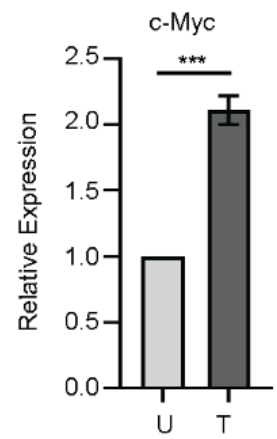

Figure 2. Expression of genes involved in mitochondrial OXPHOS increases after acute cisplatin treatment. Gene expression of the indicated genes by qPCR in OVCAR5 (A) and OVSAHO (B) cells untreated or treated with $12 \mu \mathrm{M}$ or $4 \mu \mathrm{M}$ cisplatin, respectively, for 16 hours. Expression of hexokinase isoforms in OVCAR5 (C) and OVSAHO (D) cells treated as in A and B. HIF-1a (E) and c-Myc (F) expression in OVCAR5 cells treated as in A. Graphs display mean fold change \pm SEM relative to untreated. Expression of all the genes was normalized to the house keeping gene Actin $B$. For all untreated versus cisplatin treated, $P$ values ${ }^{*}<0.05,{ }^{* *}<0.005$ and ${ }^{* \star *}<$ 0.0005 . 
A.

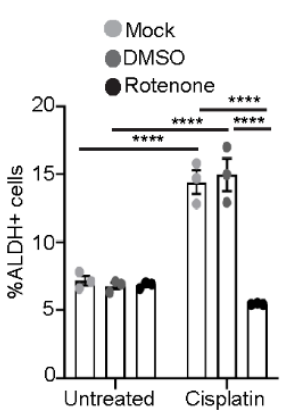

D.
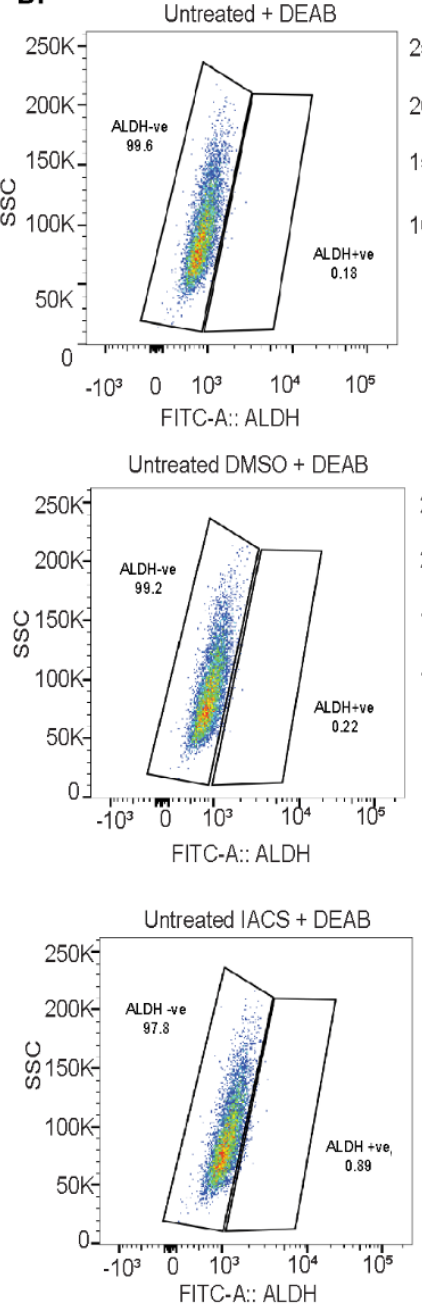

E.

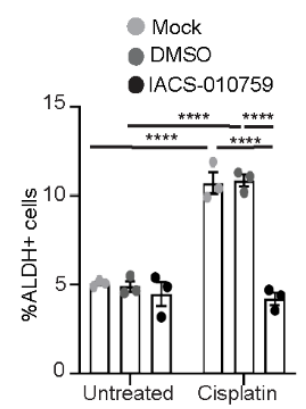

B.
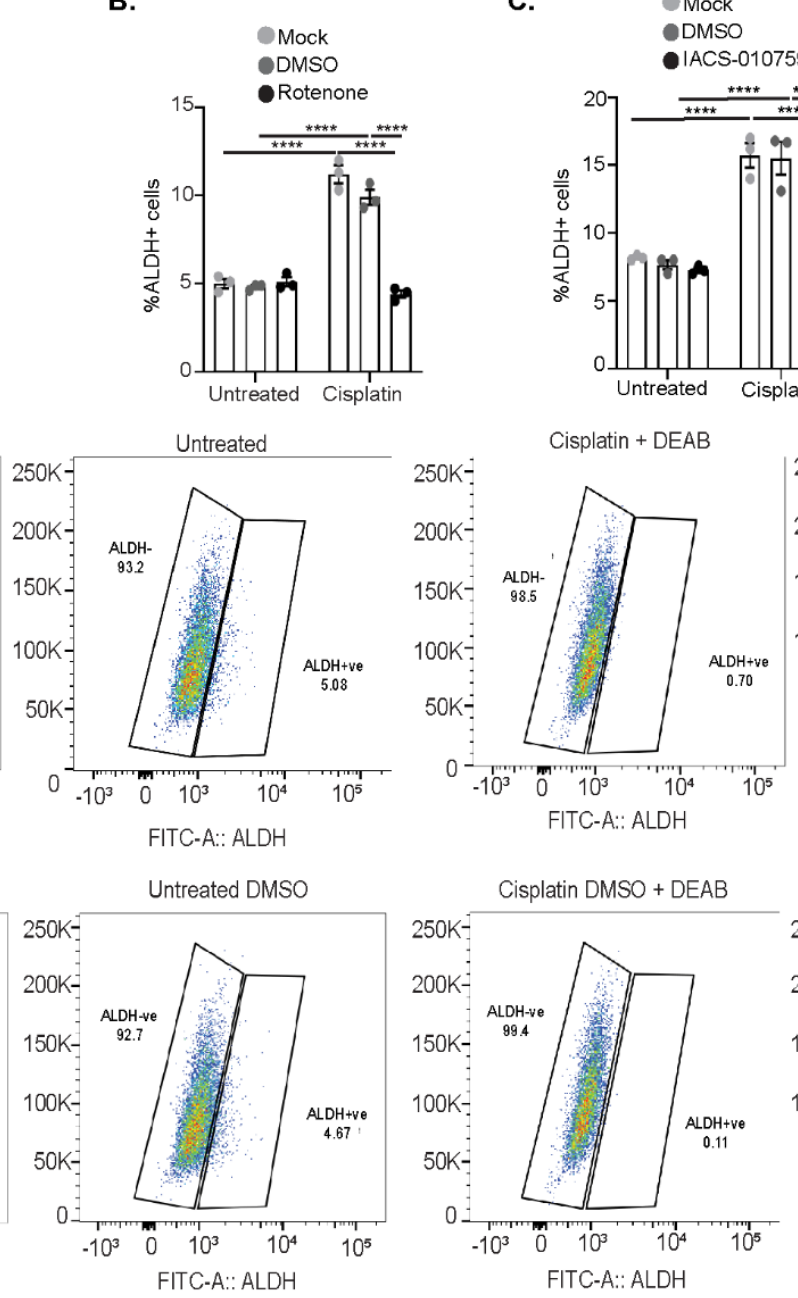

C. Mock
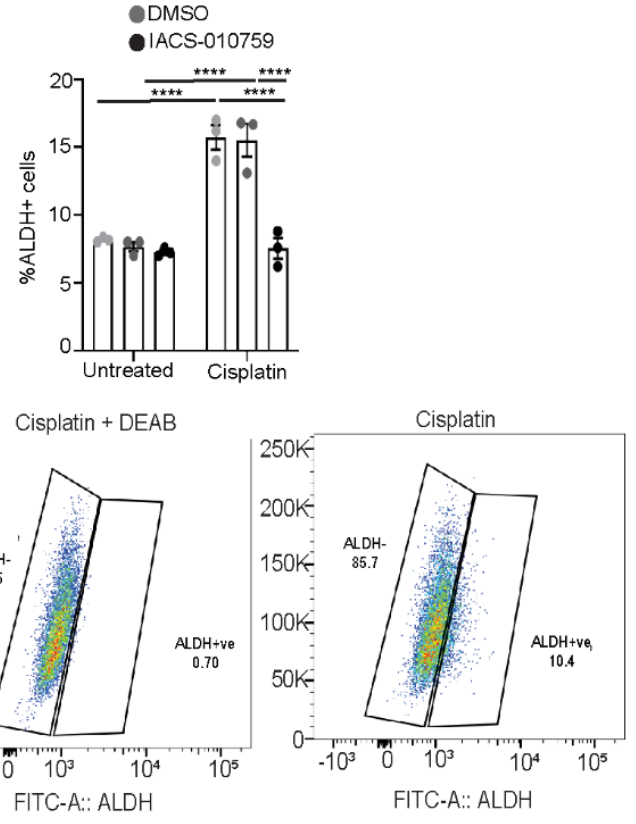

FITC-A:: ALDH
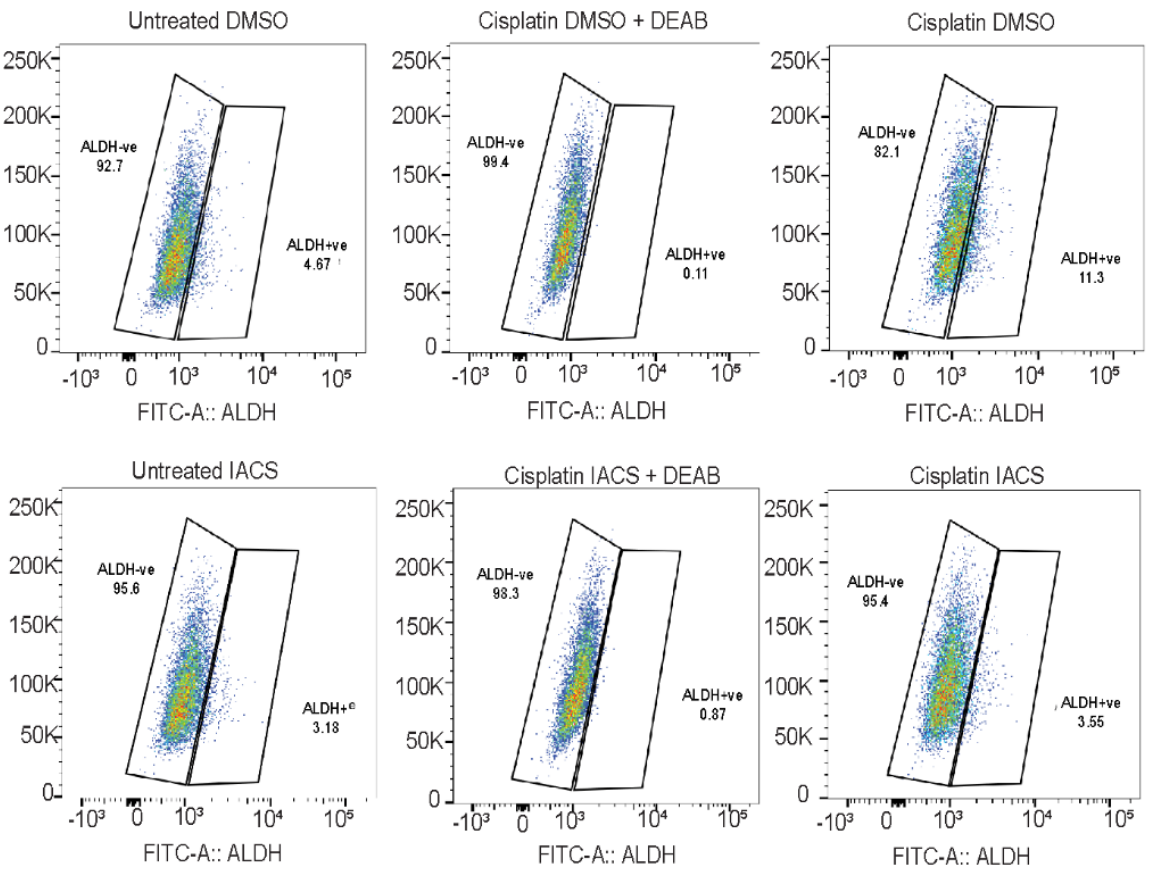

FITC-A:: ALDH
F.

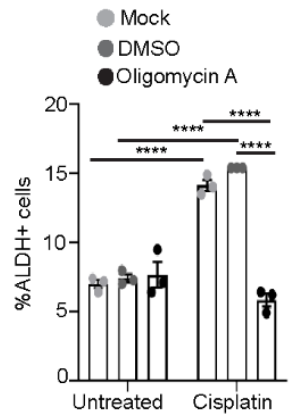


Figure 3. Mitochondrial OXPHOS inhibitors in combination with cisplatin block the platinum-induced increase in percent ALDH+ cells. Percent ALDH+ OVCAR5

(A) and OVSAHO cells (B) untreated or treated with $12 \mu \mathrm{M}$ or $4 \mu \mathrm{M}$ cisplatin, respectively, alone or in combination with DMSO or $5 \mu \mathrm{M}$ Rotenone for 16 hours followed by ALDEFLUOR assay. Percentage ALDH+ OVCAR5 (C) and OVSAHO (D-E) cells treated with cisplatin alone or in combination with DMSO or $1 \mu \mathrm{M}$ IACS-010759 for 16 hours followed by ALDEFLUOR assay. (D) shows gates used to determine ALDH+ cells using DEAB controls for one biological replicate of OVSAHO cells. (F) Percent ALDH+ OVCAR5 cells treated with cisplatin alone or in combination with DMSO or $1 \mu \mathrm{M}$ oligomycin for 16 hours followed by ALDEFLUOR assay. Graphs display mean \pm SEM percent $\mathrm{ALDH}+$ cells in $\mathrm{N}=3$ biological replicates. For all untreated versus cisplatin treated, $P$ values ${ }^{*}<0.05,{ }^{* *}<0.005,{ }^{* * *}<0.0005,{ }^{* * * *}<0.0001$. 
bioRxiv preprint doi: https://doi.org/10.1101/2022.02.01.478738; this version posted February 3, 2022. The copyright holder for this preprint (which was not certified by peer review) is the author/funder, who has granted bioRxiv a license to display the preprint in perpetuity. It is made available under aCC-BY-NC-ND 4.0 International license.

A.

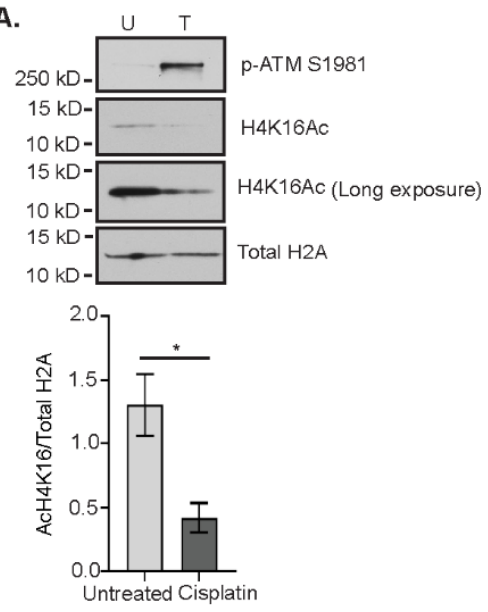

D.

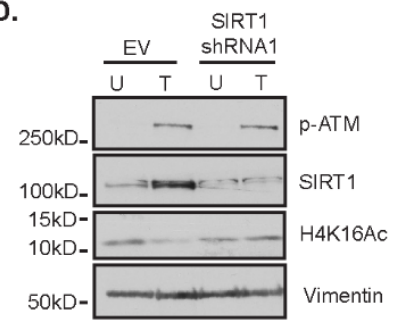

G.

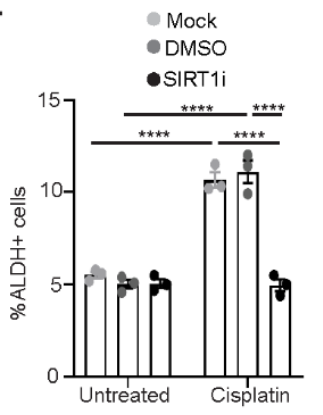

I.

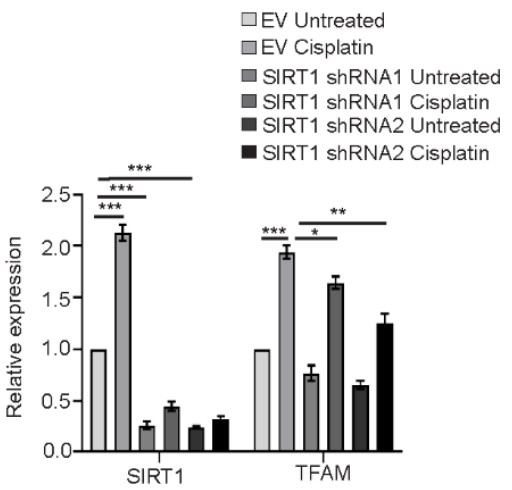

B.

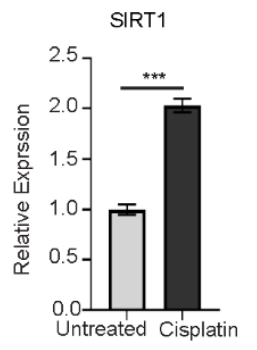

E.

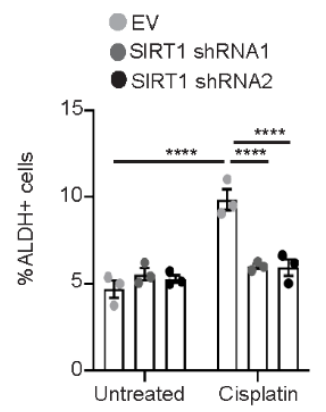

H.

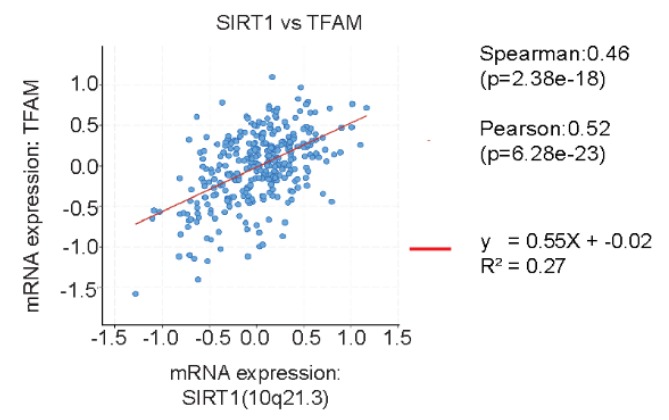

C.

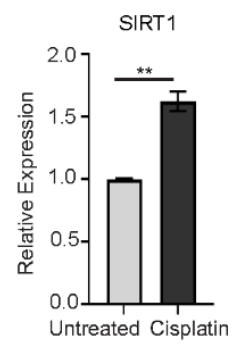

F.

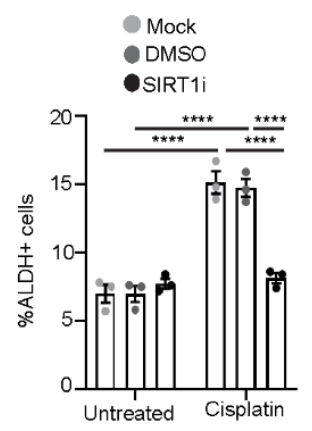


Figure 4. Platinum-induced increase in SIRT1 activity contributes to the enrichment of ALDH+ cells. (A) OVCAR5 cells were untreated (U) or treated with IC50 dose of cisplatin (12 $\mu \mathrm{M})$ for 16 hours $(\mathrm{T})$. Cell lysates were collected and analyzed by Western blot. Graph displays mean \pm SEM densitometric analysis of $N=3$ biological replicates of $\mathrm{H} 4 \mathrm{~K} 16 \mathrm{ac}$ relative to total $\mathrm{H} 2 \mathrm{~A}$. Relative expression of SIRT1 in OVCAR5 (B) and OVSAHO (C) cells untreated or treated with $12 \mu \mathrm{M}$ or $4 \mu \mathrm{M}$ cisplatin, respectively, for 16 hours. (D) OVCAR5 cells infected with empty vector (EV) or SIRT1 viral shRNA followed by treatment as in C. Lysates were collected and analyzed by Western blot for the indicated proteins. (E) OVCAR5 cells infected EV or SIRT1 viral shRNA, untreated or treated as in C followed by ALDEFLUOR assay. Graph shows mean \pm SEM percent $A L D H+$ cells in $\mathrm{N}=3$ biological replicates. Percent $\mathrm{ALDH}+$ OVCAR5 (F) and OVSAHO (G) cells treated with cisplatin alone or in combination with DMSO or $3 \mu \mathrm{M}$ Ex-527 for 16 hours followed by ALDEFLUOR assay. Graph depicts mean \pm SEM percent ALDH+ cells in $\mathrm{N}=3$ biological replicates. $(\mathbf{H})$ Correlation analysis of SIRT1 and TFAM expression using RNA-seq data from TCGA OC patient dataset. (I) Relative expression of SIRT1 and TFAM in OVCAR5 cells infected with EV or SIRT1 viral shRNA and treated as in C. Graph displays mean relative expression \pm SEM to the untreated. Gene expression was normalized to the house keeping gene Actin $B$. For all comparisons, $P-{ }^{*}<0.05,{ }^{* *}<0.005,{ }^{* * *}<0.0005,{ }^{* * *}<0.0001$. 


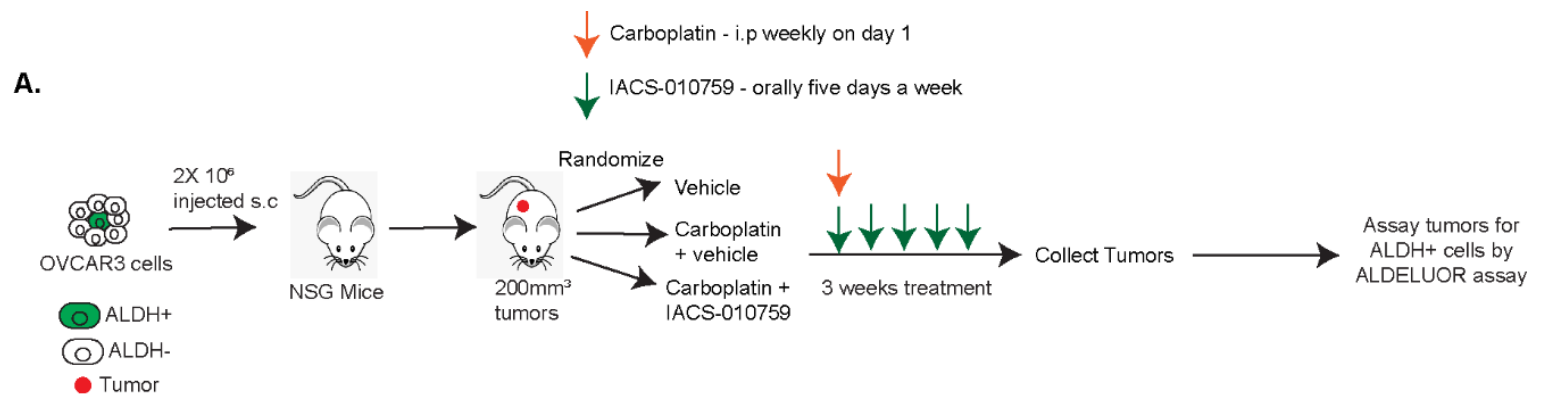

B.

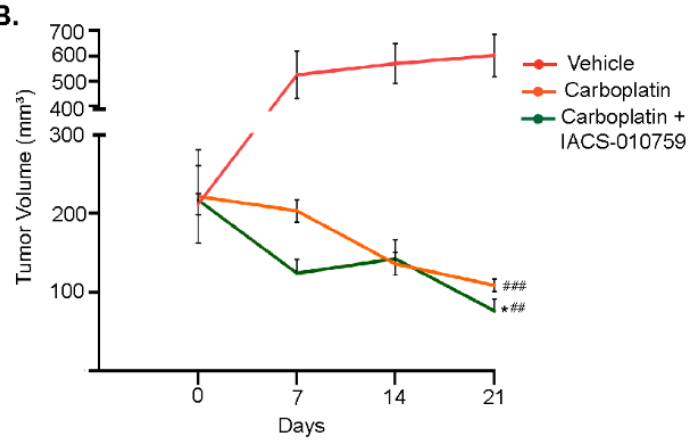

C.

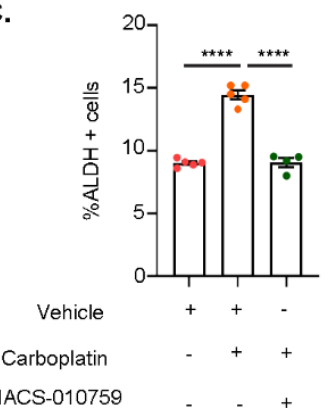

Figure 5: OXPHOS inhibition blocks the platinum-induced enrichment of ALDH+ cells in vivo

(A) $2 \times 10^{6}$ OVCAR3 cells were injected s.c in 6-7 week old NSG mice. Once the established tumors were $>100 \mathrm{~mm}^{3}$, mice were randomized into three groups and treated with vehicle alone or combination of vehicle + carboplatin or IACS-010759 for 3 weeks as indicated. At the end of the study, tumors were collected, dissociated into single cells and the ALDEFLUOR assay was performed. (B) Tumor volumes were measured using a digital caliper through 3 weeks of treatment. $\mathrm{N}=4-5$ mice per group. ${ }^{*} P$ relative to carboplatin + vehicle. \#P relative to vehicle. (C) Percentage of $A L D H+$ cells in dissociated xenograft tumor samples using ALDEFLUOR assay. Graph indicates mean $\pm S E M$ ALDH percentage in the different treatment groups. $N=4-5$ mice per group. For all treated with vehicle alone versus carboplatin + vehicle or IACS010759, $P$ values ${ }^{*}<0.05,{ }^{* *}<0.005,{ }^{* * *}<0.0005,{ }^{* \star * *}<0.0001$ 